AperTO - Archivio Istituzionale Open Access dell'Università di Torino

\title{
How Can Gastric Cancer Molecular Profiling Guide Future Therapies?
}

\section{This is the author's manuscript}

Original Citation:

Availability:

This version is available http://hdl.handle.net/2318/1565043

since 2016-08-29T11:33:32Z

Published version:

DOI:10.1016/j.molmed.2016.05.004

Terms of use:

Open Access

Anyone can freely access the full text of works made available as "Open Access". Works made available under a Creative Commons license can be used according to the terms and conditions of said license. Use of all other works requires consent of the right holder (author or publisher) if not exempted from copyright protection by the applicable law. 


\section{(2) \\ UNIVERSITÀ DEGLI STUDI DI TORINO}

This is an author version of the contribution published on:

Questa è la versione dell'autore dell'opera:

[Trends in Molecular Medicine, volume 7, issue 22, 2016,

DOI: 10.1016/j.molmed.2016.05.004]

The definitive version is available at:

La versione definitiva è disponibile alla URL:

[http://www.sciencedirect.com/science/article/pii/S1471491416300284] 


\title{
How Can Gastric Cancer Molecular Profiling Guide Future Therapies?
}

\author{
Simona Corso ${ }^{1}$, Silvia Giordano ${ }^{1}$ \\ 1 University of Torino, Department of Oncology, Candiolo Cancer Institute-FPO, IRCCS, Str. Provinciale 142, 10060 Candiolo, Torino, Italy
}

Gastric cancer is the third greatest global cause of cancer-related deaths. Despite its high prevalence, only recently have comprehensive genomic surveys shed light on its molecular alterations. As surgery is the only curative treatment strategy and chemotherapy has shown limited efficacy, new treatments are urgently needed. Many molecular therapies for gastric cancer have entered clinical trials but-apart from Trastuzumab and Ramucirumab-all have failed. We analyze the current knowledge of the genetic 'landscape' of gastric cancers, elaborating on novel, preclinical approaches. We posit that this knowledge lays the basis for identifying bona fide molecular targets and developing solid therapeutic approaches, requiring accurate patient selection and taking advantage of preclinical models to assist clinical development of novel combination strategies.

\section{Trends}

Gastric cancer is the third leading cause of cancer mortality worldwide. Surgery is the only curative treatment strategy and conventional chemotherapy has shown limited efficacy, with a median overall survival of 10 months.

Trastuzumab and Ramucirumab (targeting HER2 and VEGFR, respectively) are currently the only gastric cancer approved targeted therapies; hence, new treatments are urgently needed.

Despite the high prevalence of gastric cancer, very few comprehensive genomic surveys have been performed to date.

Recently, the Cancer Genome Atlas (TCGA) Research Network and the Asian Cancer Research Group (ACRG) separately reported two Gastric Cancer whole genome molecular profiling analyses, proposing two new molecular classifications for gastric malignancies.

Given that the use of targeted therapies in gastric tumors is much less common than in other cancers, the reported data represent a critical starting point to design more appropriate clinical trials based on the principles of precision medicine.

Starting from available molecular data, recent preclinical models (tumor-derived cell lines, organoids, and PDXs) should complement clinical investigations, so as to facilitate the development of targeted therapeutic approaches.

\section{Keywords}

gastric cancer; targeted therapies; HER2; tyrosine kinase receptors

\section{Pathological Classifications of Gastric Carcinomas}

In spite of decreasing incidence and mortality in the past decades, gastric cancer still remains one of the most common causes of cancer-related death [1]. Globally, gastric cancer accounts for 989600 new cases and 738000 deaths each year, with a case fatality ratio of 0.75 [2]. Incidence is strongly influenced by ethnic and geographical factors: it is higher in Eastern Asia, Eastern Europe, and South America, while North America and Africa show the lowest rates [3]. In Europe, stomach cancer is the fifth most common cancer with 159900 new cases and 118200 deaths reported in 2006 [4].

Epidemiologically, dietary factors and Helicobacter pylori (see Glossary) infection are among the major risk factors for the development of distal tumors, while those for proximal cancers include gastroesophageal reflux disease and obesity [5]. 
Proximal/gastroesophageal junction tumors are usually associated with inflammation due to chronic gastric acid/bile reflux. Inflammation is characteristically absent in the development of gastric cancer resulting from germline mutations in the human cadherin 1 gene (CDH1) [6]. A role for the Epstein-Barr virus (EBV; whose genome can be identified in tumor cells) has also been demonstrated [7] (Box 1). Approximately $80-90 \%$ of gastric carcinomas develop in a sporadic setting, and the remaining show familial clustering, with approximately 1-3\% exhibiting a clear inherited genetic susceptibility [6].

\section{Box 1.}

\section{Role of Epstein-Barr Virus in Epithelial Tumors}

EBV-associated epithelial cancer (characterized by the presence of an integrated EBV genome) represents approximately $80 \%$ of EBV-associated neoplasms [55]. The most frequent neoplasms are nasopharyngeal carcinoma $(89 \%)$ and gastric cancer (10\%). EBV is not sufficient to induce the full malignant transformation but is believed to impart the first 'hit' to the process. In fact, the presence of a clonal EBV genome in tumor cells indicates that viral infection takes place at the beginning of the tumorigenic process. P16 inactivation (commonly found in EBV+ tumors) is required to allow persistent EBV inactivation in epithelial cells, eliciting clonal expansion of EBV-infected cells [56]. Another common event in EBV+ tumors is PDL1/2 overexpression that underlines the role of immune evasion for progression of these tumors. Finally, EBV+ tumors are rarely associated with p53 mutations, which are otherwise very common in EBVtumors of the same histotype [11].

Two main classifications are used to define gastric adenocarcinomas: (i) the World Health Organization (WHO) classification recognizes four histological subtypes (papillary, tubular, mucinous, and poorly cohesive) and (ii) the Lauren classification identifies intestinal, diffuse, or mixed subtypes [8]. Neither the WHO nor the Lauren classification systems are particularly clinically useful, as their prognostic and predictive capabilities cannot adequately guide patient management. Therefore, at present, the histopathological, anatomical, and epidemiological distinctions are not taken into account in the clinical management of the disease, either initially for potentially curative treatment, or, palliatively, for advanced disease.

While the identification of specific molecular phenotypes in other epithelial malignancies has had profound implications for treatment strategies, this has had a much lower impact on gastric cancer. As molecular alterations of drug-targeted genes are fairly frequent 9 and 10 , research performed in preclinical models could help identify actual tumor drivers along with the best therapeutic options.

\section{TCGA (The Cancer Genome Atlas) Molecular Characterization of Gastric Adenocarcinomas}

Recently, owing to new technological platforms, molecular landscapes of gastric cancer have been explored and two new classifications have been proposed [11]. The TCGAResearch Network has characterized 295 primary gastric adenocarcinomas using six molecular platforms (evaluating somatic copy number alterations, whole exome sequencing, mRNA and miRNA sequencing, DNA methylation analysis, and phosphoproteomics). Their integrated analysis has allowed the identification of four molecularly distinct subtypes (Figure 1). 


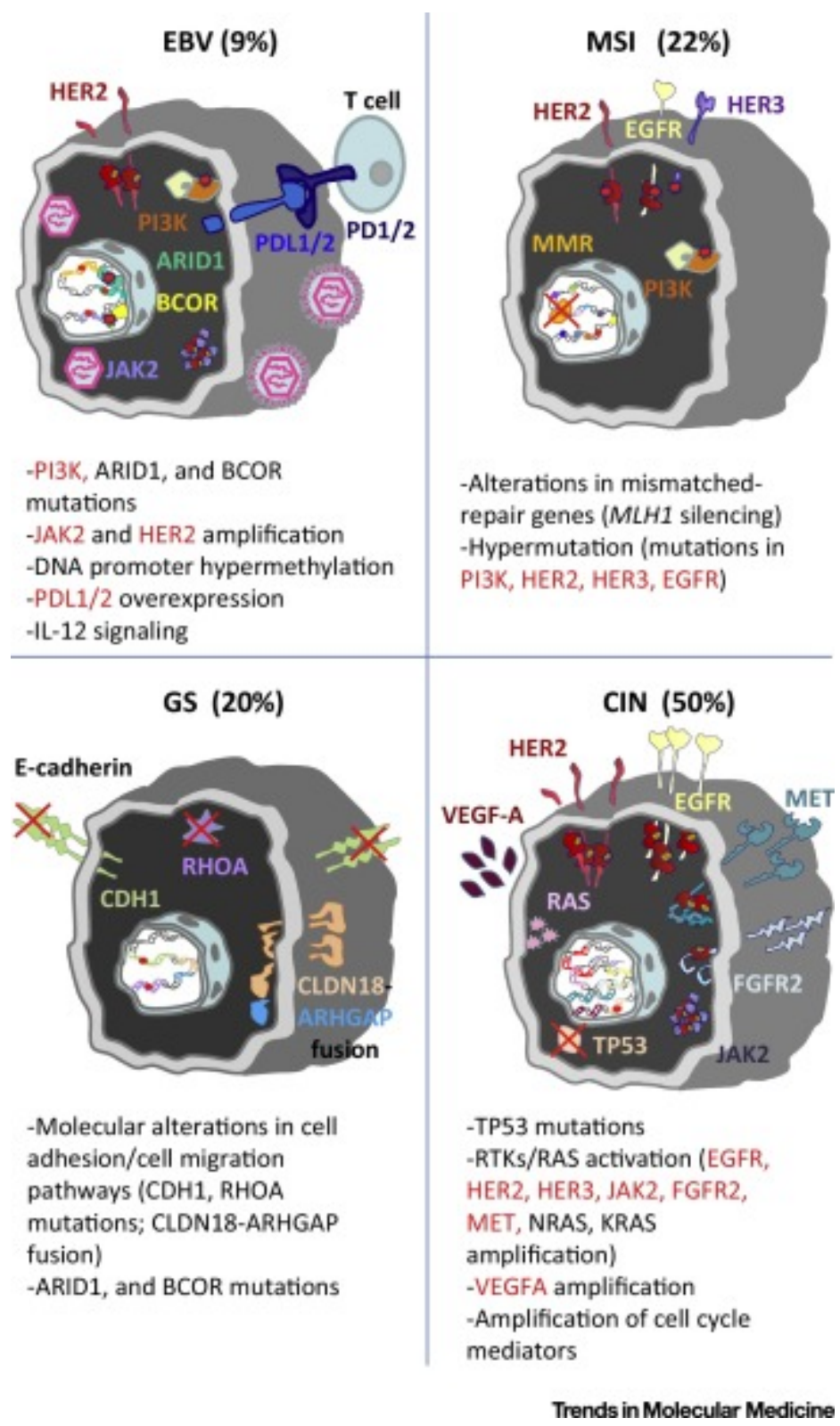

Figure 1. Gastric Cancer Classification Proposed by the Cancer Genome Atlas Research Network. EBV, tumors displaying EpsteinBarr virus positivity; MSI, tumors with microsatellite instability; GS, tumors genomically stable; CIN, tumors with chromosomal instability. Major molecular alterations are depicted. Currently 'druggable' targets are highlighted in red.

The first group ( $9 \%$ of gastric adenocarcinomas) was significantly enriched in EBV burden and was characterized by extensive DNA promoter hypermethylation (typically associated with silencing of specific genes). Interestingly, the methylation profile was different from that observed in the microsatellite instability (MSI) subtype, with several genes differentially silenced [e.g., MLH1 (Mutl homolog 1), a key component of the DNA mismatch repair system, which is hypermethylated in MSI tumors but not in EBV+ tumors]. Interestingly, the EBV subtype showed the highest frequency of PIK3CA(phosphatidylinositol-4,5-bisphosphate 3-kinase catalytic subunit a) mutations (80\% of cases), dispersed along the sequence and common to those present in the TCGA dataset or in the COSMIC (Catalogue Of Somatic Mutations In Cancer) repository, in $68 \%$ of the cases [11]. Other common alterations or mutations were found in ARID1A(AT-rich interaction domain $1 \mathrm{~A}$ gene, 55\%) and in BCOR (BCL6 corepressor gene, 23\%), plus the amplification of a locus containing JAK2 (Janus kinase) and PDL1/2 (Programmed cell death 1 ligand 1 and 2; inhibitory immune checkpoint) genes (15\%). This latter observation can be functionally coupled with the finding that strong IL-12 mediated signaling molecular signatures were identified, suggestive of a robust presence and/or communication/with 
immune cells in these biopsies. Consequently, targeting PI3K and inhibitory immune checkpoints may indeed yield important therapeutic targeting options.

The second group was enriched for MSI (22\% of gastric adenocarcinomas), presenting elevated mutation rate and hypermethylation [11]. Mutations $\quad$ of $\quad$ kinases as EGFR(5\%), HER2 (5\%), HER3 (14\%), JAK2 (11\%), FGFR2 (2\%), MET (3\%), and PIK3CA(42\%) were present. Interestingly, $\mathrm{MSI}$ tumors revealed common alterations in major histocompatibility complex class I genes, such as B2M and HLA-B, potentially suggesting reduced tumor antigen presentation to cells of the immune system.

The third group, named genomically stable (20\% of cases), lacked extensive somatic copy number aberrations, and biopsies often presented diffuse type histology. This subtype frequently presented alterations in genes involved in cell adhesion, such asRHOA (15\%), CDH1 (26\%), and CLDN18/ARHGAP (15\%). Moreover, these tumors also exhibited elevated expression of cell adhesion and angiogenesis-related pathways.

The last group was defined as CIN, characterized by chromosomal instability (50\% of cases) [11]. From a molecular point of view, among the non-hypermutated tumors, CIN cancers showed the highest frequency of p53 mutations. However, an important distinction for these was the activation of the RTK/RAS pathway as a consequence of gene amplification. Indeed, in CIN tumors they found amplification of EGFR (10\%),HER2 (24\%), HER3 (8\%), JAK2 (5\%), FGFR2 (8\%), MET (8\%), PIK3CA (10\%), andKRAS/NRAS (18\%).

Overall, the authors of this work did not find systematic differences in the distribution of subtypes between patients of East Asian and Western origin. As the identified tumor types were not linked to different prognoses, this classification may not substantially help physicians from a prognostic point of view, but the findings, however, are of outstanding importance; they may allow the identification of putative and useful target therapies to improve the yet unsatisfactory outcome of gastric cancer patients.

\section{ACRG (Asian Cancer Research Group) Molecular Characterization of Gastric Adenocarcinomas}

The ACRG recently provided a new classification of gastric cancer, identifying four subtypes [12] (Figure 2): MSI (displaying loss of MLH1); MSS/EMT (microsatellite stable and epithelial-mesenchymal transition; displaying loss of cadherin); MSS/TP53+ (p53 active) and MSS/TP53- (p53 inactive). One of the most important aspects of this classification is that it correlates the molecular subtypes with clinical prognosis. The MSS/EMT subtype occurred at a younger age, was mainly of the diffuse type, was diagnosed at late stages, and showed the worst prognosis. The MSI subtype occurred mainly in the antrum (75\%), was preferentially an intestinal subtype (>60\%), was mostly diagnosed at early stages, and showed the best prognosis. EBV positivity occurred more frequently in the MSS/TP53+ group. MSS/TP53+ and MSS/TP53- presented an intermediate prognosis. Moreover, the MSS/EMT type was associated with a higher chance of recurrence compared with MSI (63\% versus 23\%). The first site of recurrence was different according to the subtype: peritoneal seeding was more frequent in the MSS/EMT (64\% versus $23 \%$ ), and liver-limited metastases were more frequent in the MSI (23\%) and MSS/TP53- subtypes (21\%). Concerning the molecular status of different subtypes, the MSI subtype was associated with the presence of hypermutations in genes such as KRAS (23\%), ALK (16.3\%), ARID1A (44.2\%), and those involved in the PI3K pathway (42\%). The MSS/EMT subtype presented a lower mutation frequency compared with the other MSS groups $(P<0.001)$. Within this category, the MSS/TP53- subtype exhibited the highest prevalence of TP53 mutations (60\%) while the MSS/TP53+ presented a higher frequency of mutations in APC, ARID1A, KRAS,PIK3CA, and SMAD4. Considering the copy number profile, genomic instability was present in $28 \%$ of all cases and was significantly associated with the MSS/TP53-subtype[12]. 


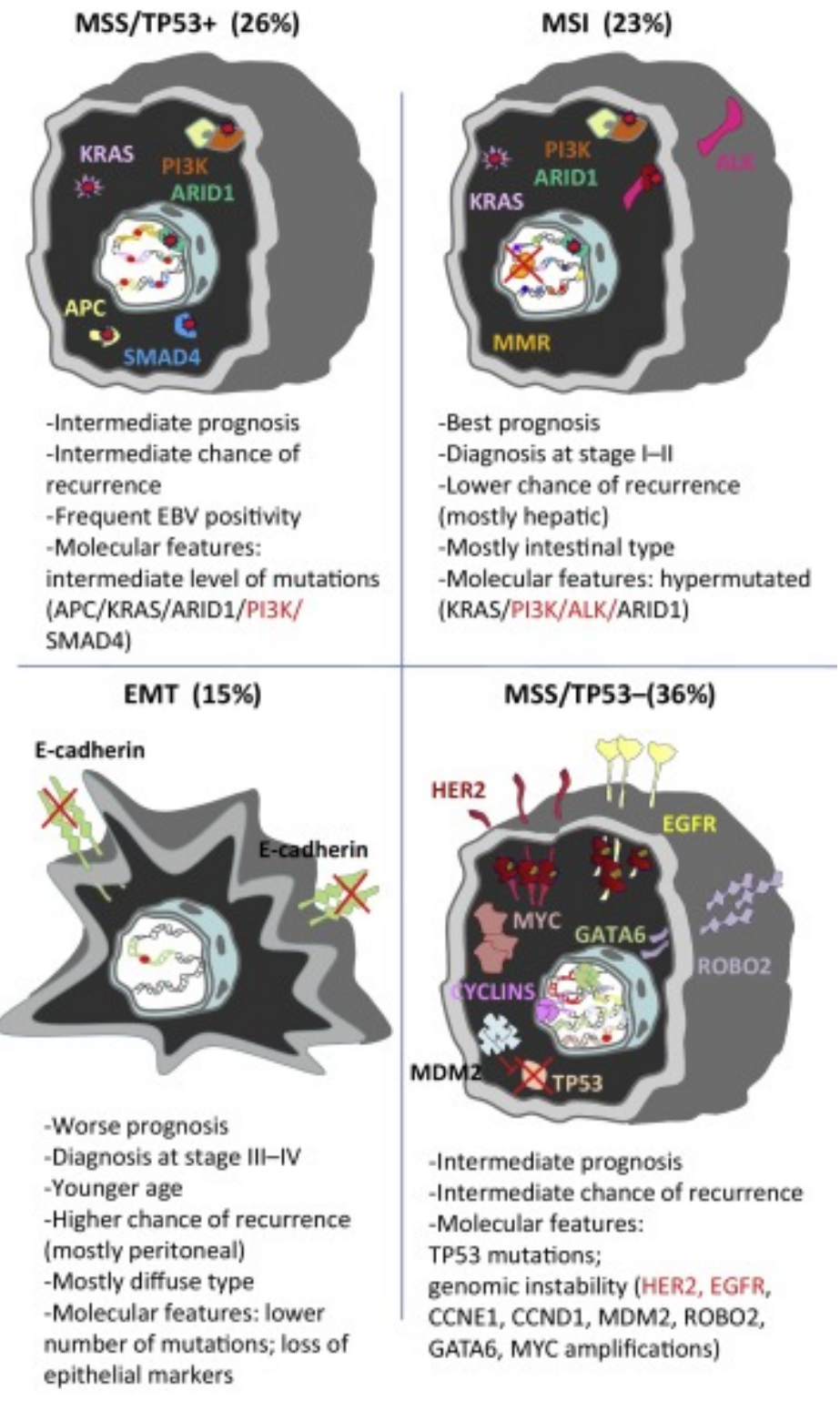

Trends in Molecular Medicine

Figure 2. Gastric Cancer Classification Proposed by the Asian Cancer Research Group. MSI, tumors with microsatellite instability; EMT, tumor with epithelial-mesenchymal transition; MSS/TP53+, tumors with microsatellite stability and p53 activity; MSS/TP53-, tumors with microsatellite stability and loss of p53 activity. Major molecular alterations and prognostic value are depicted. Currently 'druggable' targets are highlighted in red.

\section{Comparison between TCGA and ACRG Classifications}

A comparison between the TCGA and the ACRG classifications shows similarities and differences. Both identified a MSI subtype characterized by high mutation frequency and best prognosis; TCGA GS, EBV+, and CIN subtypes were enriched in ACRG MSS/EMT, MSS/TP53+, and TP53-, respectively. However, CIN and GS TCGA tumors were present across all ACRG subtypes. On the basis of the different frequency of CDH1 andRHOA mutations, the TCGA GS subtype was not equivalent to the ACRG MSS/EMT subtype. Similarly, MSS/TP53 did not overlap with the TCGA EBV subtype, as only 12/18 EBV+ tumors were present in the MSS/TP53 group (79 tumors). Why are these two classifications only partially overlapping? Possible explanations are that: (i) a larger proportion of the ACRG tumors constituted the Lauren diffuse type, and fewer constituted proximal or junctional ones, as it is known that these tumors are very different in terms of their behavior and evolution; (ii) the ethnic origin of the patients is different: they are mainly from Korea in the ACRG and from USA and Western Europe in the TCGA; and (iii) the two groups used different technological platforms. 


\section{Molecular Classifications of Gastric Adenocarcinomas: What Can They Teach Us?}

These two classifications, while not completely overlapping, represent an important step forward in advancing our knowledge of the molecular basis and subtyping of gastric cancer. As previously discussed, it may provide fundamental help in the development of rational molecular therapies. To date, conventional therapies rely mainly on surgery, chemotherapy, and radiotherapy, but the available cytotoxic agents are applied indiscriminately to all subtypes-with only modest success. Concerning molecular therapies, only two target therapies have been approved so far by the FDA and the European Union for advanced gastric cancer: Trastuzumab (anti-HER2 monoclonal antibody, mAb) and Ramucirumab [an anti-vascular endothelial growth factor receptor 2 (VEGFR-2) mAb, with antiangiogenic activity] 13, 14 and 15. However, most patients have seen limited benefit from these drugs. Moreover, trials with other anti-HER2 or antiangiogenic drugs have recently failed 16, 17, 18 and 19 (Figure 3).

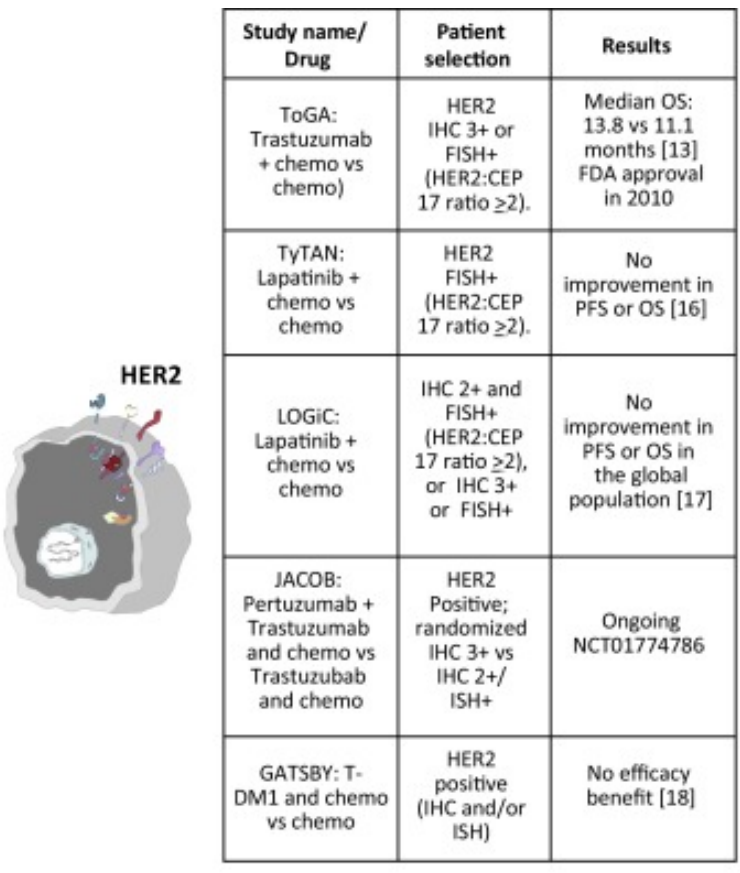
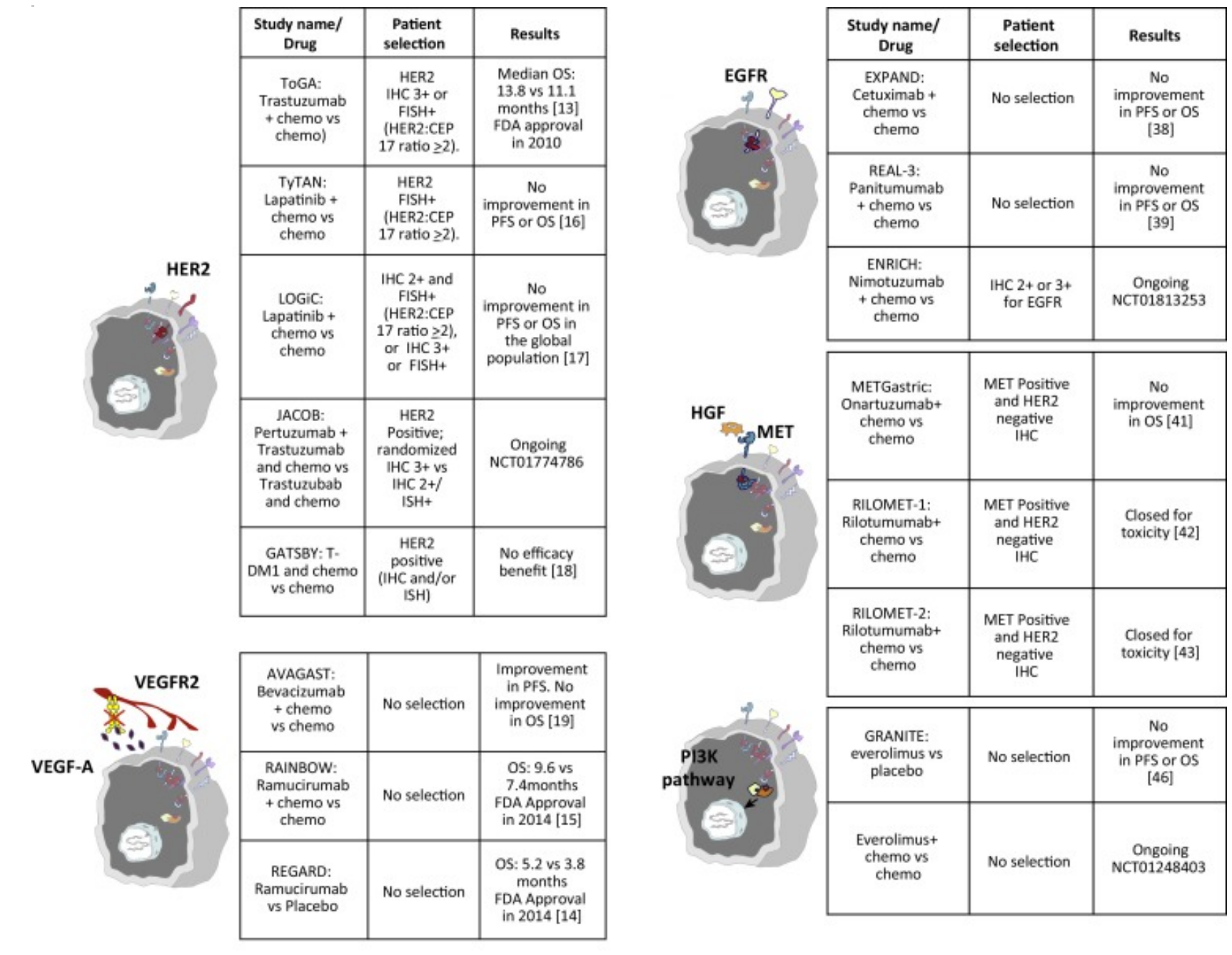

Trends in Molecular Medicine

Figure 3. Phase III Clinical Trials with Molecular Therapies in Gastric Cancer. For each clinical trial, the criteria for patient selection and the main results are summarized 13,16,17 and 18. Abbreviations: IHC, immunohistochemistry; FISH, fluorescent in situ hybridization; ISH, in situ hybridization; CEP, centromere probe; OS, overall survival; PFS, progression-free survival; FDA, Food and Drug Administration (USA).

Despite the diverse molecular alterations identified in gastric carcinomas, from the perspective of metastasis, the only molecular therapy currently approved for metastatic gastric cancer (based on tumor genetic alterations) is Trastuzumab [13]. Even though results obtained in Trastuzumab clinical trials suggest that HER2 is a bona fide target in gastric cancer, the efficacy of the treatment is not durable [13]. An improvement could come from a rigorous selection of 
patients. Many preclinical studies have shown that cancer cells can be 'addicted' to genetically altered oncogenes [20]. The most frequent causes of addiction to activated receptor tyrosine kinases are mutations and amplifications [21]. In particular, in the case of HER2, an amplification of at least 4-fold (i.e., eight HER2 copies) is required to observe a clinical response to anti-HER2 therapies: the higher the level of amplification, the stronger the patient's response [22]. Moreover, it is still unclear if tumors displaying HER2 activating mutations (2-3\% of gastric cancers) do respond to Trastuzumab and should thus be treated. Overall, predictive biomarkers of response to HER2 are still missing and no mechanisms of primary or secondary resistance to Trastuzumab-which would allow patient exclusion-have been identified in gastric cancer. As an example, the effect on response to therapy from the co-presence of mutations/amplifications of other RTKs, PIK3CA, and KRAS (all identified in the described reports) has yet to be evaluated.

How could we address the search of negative and positive predictors of response? As already performed in the case of other tumors, the use of patient-derived xenograft(PDX) models could help in this quest (Figure 4, Box 2 ). For example, in colon cancer, Bertotti et al. studied a wide cohort of metastatic colon cancer PDXs and evaluated the response to treatment with the anti-EGFR mAb Cetuximab [23]. Response to Cetuximab in PDXs paralleled that reported for colon cancer patients. Indeed, PDXs showing mutations in KRAS, NRAS, BRAF, or PI3K were insensitive to Cetuximab. Analyzing non-responder PDXs, the authors identified HER2 amplification and mutations as mechanisms of resistance to anti-EGFR therapy 23 and 24 . These data led to the undertaking of a Phase II clinical trial in metastatic colon patients displaying HER2 amplifications, which showed the efficacy of HER2 targeting (30\% objective responses, 59\% disease control, in chemotherapy and anti-EGFR mAb refractory patients) [25]. As the generation of gastroesophageal PDXs is feasible 26, 27 and 28 and gastric PDX platforms are available 29 and 30, the analysis of responder and non-responder HER2+ tumors could shed light on the mechanisms of Trastuzumab resistance and facilitate the identification of response predictors to gastric cancer. One alternative and/or complementary approach to the analysis of PDXs would be the in vitro use of tumor-derived cells and tumor organoids to verify the sensitivity of tumor cells to different targeted therapies $31,32,33,34$ and 35 . This would provide the important advantage of allowing large-scale drug screens in a shorter timeframe [36] (Figure 4).

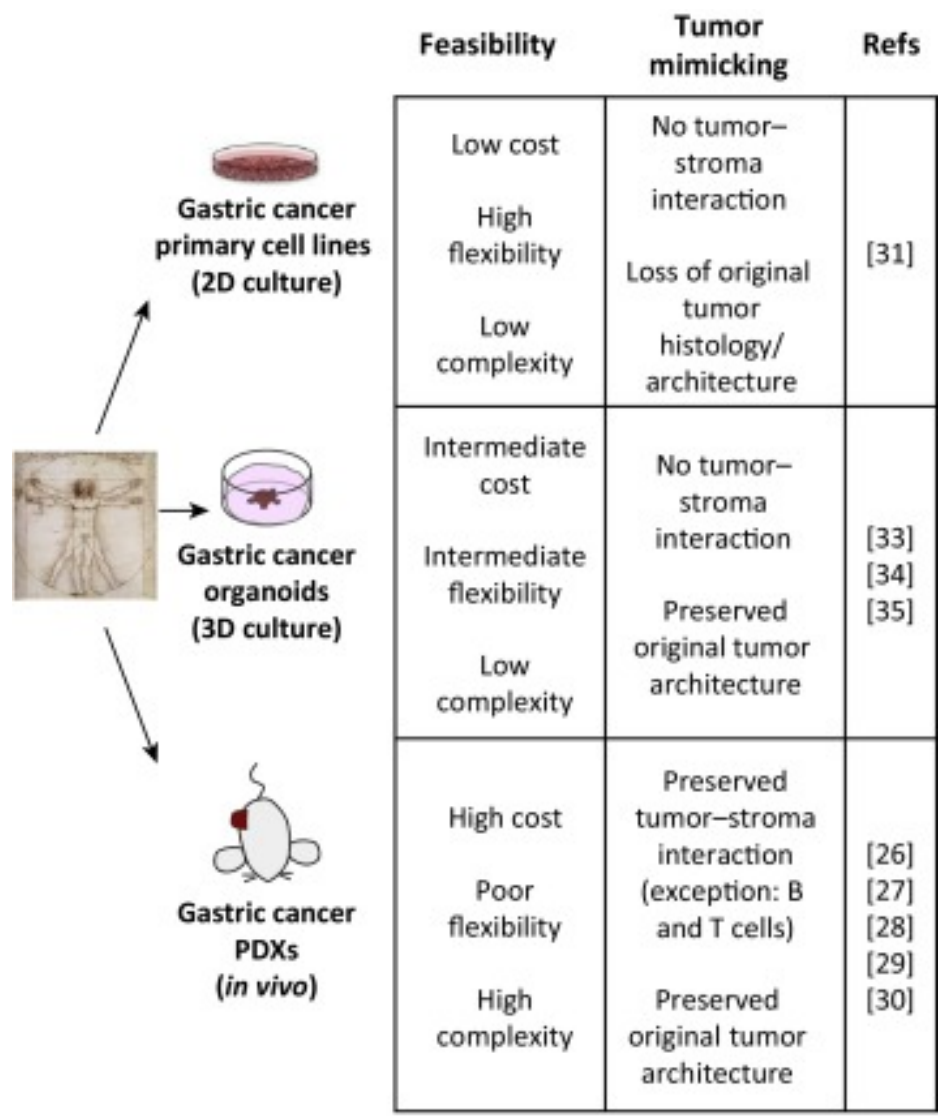


Figure 4. Patient-Derived Preclinical Models of Gastric Cancer. The advantages and disadvantages for each model are summarized; recent studies using these models to investigate the role of different targets, or the response to therapies in gastric cancer are also listed 26, 27, 28, 29, 30, 31, 33, 34 and 35.

\section{Box 2.}

\section{PDX Models in Translational Cancer Research}

PDX models are increasingly used for drug screening, biomarker discovery, and preclinical testing of personalized medicine approaches. Many studies have indeed shown that PDXs closely recapitulate the histological, molecular, and biological features of the tumors from which they were derived [57]. The use of PDXs facilitates nonconventional prospective trials ex vivo and entail the subcutaneous implantation of fresh tumor biopsies in immunocompromised mice, followed by the establishment of perpetuated 'cancer lines' in experimental animals. Moreover, PDXs facilitate combining therapeutic treatments, allowing to dynamically monitor a 'clinical response'. This in turn enables researchers to derive molecularly based response signatures for targeted therapies. By exploiting a PDX colony large enough to mirror the heterogeneity of gastric cancer genotypes (including low prevalence ones), it is possible to validate known potential actionable targets and generate hypotheses for proof-of-concept precision medicine trials in patients. The use of clinically annotated PDXs as a hypothesis generation tool couples the higher flexibility of preclinical analysis with the informative value of population-based studies.

Another interesting possibility derived from these studies is that in different tumor contexts, effective targeting of the same molecule might require diverse therapeutic approaches. Indeed, anti-HER2 monotherapy (with Trastuzumab, Pertuzumab-two mAbs-or Lapatinib-a dual EGFR/HER2 tyrosine kinase inhibitor) is efficacious in inhibiting HER2 in breast cancer [37], but studies performed in PDXs have shown that it is poorly effective in colon cancer, where a good response might be obtained only with the combination of an anti-HER2 mAb and Lapatinib [23]. Recently obtained data in human patients reinforce this finding [25]. Along these lines, different approaches to target HER2 could also be used in HER2-amplified gastric PDXs or in cell lines/organoids, to identify the best therapeutic approach.

As previously mentioned, various RTKs are genetically altered in gastric cancer, but their clinical targeting is not yet approved. In fact, Phase II and III clinical trials targeting EGFR, MET, and FGFR2 have returned disappointing results $38,39,40,41,42,43$ and 44 (Figure 3). However, in most cases, recruited patients have not been selected based on genetic alterations of the investigated target. This becomes a critical point to consider if the mutated target of interest is present at a very low frequency in the subjects (unselected population), because the number of response outcomes could be too low to be statistically significant. Moreover, many of the performed trials have not reported anex post analysis, which would allow investigating if the few responders share genetic features. While the identification of mutations is not questionable, the functional definition of 'gene amplification' is less obvious and depends on the utilized technique and chosen threshold. At the moment, with the exception of HER2, there is no agreement on the criteria to be used to evaluate gene amplification/overexpression. Indeed, different studies have utilized either immunohistochemistry, real-time PCR, or fluorescent in situhybridization and have applied diverse thresholds/criteria of positivity, even when using the same technique. The lack of standardized criteria makes it extremely difficult (if not impossible) to define the frequency of these amplifications and to correctly select patients that could benefit from therapies targeting amplified targets. As shown in the clinic for HER2, as well as in preclinical studies for other RTKs, tumor cells become 'addicted' to an oncogene when they have at least eight copies of that gene 22 and 45 . Thus, this is the number of gene copies a tumor should display to effectively respond to a targeted therapy [22]. However, inclusion criteria have often been less restrictive, sometimes simply based on protein overexpression without any copy number analysis. Similar negative 
results have been observed in Phase II and III clinical trials targeting the PI3K pathway performed on unselected patients 46 and 47 . From the TCGA study discussed here, we know that $72 \%$ of EBV+ and $42 \%$ of MSI+ gastric tumors presentPIK3CA mutations. Consequently, the TCGA tumor characterization criteria could facilitate the identification of PIK3CA mutated tumors. Of note, it is presently unclear which of the mutations are 'real drivers'; also in this case, the use of in vitro tumor-derived material (cells and organoids) or PDXs could be helpful in identifying the oncogenic potential of such mutations, in addition to optimizing a conceived therapeutic approach. Furthermore, since the PI3K/AKT/mTOR pathway can be inhibited at different levels of the cascade, these preclinical systems might allow the testing of different drugs and combinations, and the subsequent identification of the most efficacious ones. Finally, the use of MAPK inhibitors in KRAS mutated/amplified tumors (which represent around 17\%[11]) has not yet been explored.

The in-depth analysis of genomic data might also clarify if, in selected subgroups, it is possible to identify 'trunk genetic alterations'-that is, alterations that have appeared in early clonal tumor progenitors and thus are ubiquitously present in neoplastic cells (Box 3). Clinical experience has shown that when trunk mutations are targeted, as in the case of chronic myelogenous leukemia, the efficacy of the selected molecular therapy in strongly increased [48].

\section{Box 3.}

\section{Trunk Mutations and Intratumor Heterogeneity}

Sequencing of many tumors of the same histotype has proved the existence of genetic intertumoral heterogeneity. Moreover, genomic analysis of different samples obtained from diverse regions of a single tumor, in addition to single cell genomic analysis, have revealed the presence of intratumoral genetic heterogeneity. This has provided the opportunity of modeling tumor evolution within a 'tree structure' of tumor development. The 'trunk' of the tree is represented by somatic alterations driving tumor growth, already present in early clonal progenitors and likely to represent ubiquitous events occurring at all sites within a tissue. By contrast, somatic alterations occurring later on, following a branched separation of subclones, epitomize heterogeneous events [58]. Such heterogeneity can be observed not only inside a tumor but also at metastatic sites. Targeting 'trunk' mutations, ubiquitously present in tumor cells, may be more effective and provide new approaches to limit the onset of tumor resistance. However, 'branch' mutations help explain how tumors can evade cancer therapeutics; as these mutations can lead to activation/inactivation of pathways conferring resistance to treatment, cells bearing these mutations can be selected during treatment and become the prominent population within the tumor [59].

Finally, immunotherapy could be potentially promising in two subgroups, namely EBV+ and MSI+ tumors. Indeed, the high number of mutations present in MSI+ tumors creates neoantigens that influence the patients' response to immune checkpoint inhibitors 49 and 50 . This has been recently shown for colon cancer (restricted to MSI +) and lung cancer patients, where the response to immune checkpoint inhibitors (such as the CTLA4 mAb Ipilimumab, or the PD-1 mAbs Pembrolizumab and Nivolumab) led to detectable numbers of $T$ cells recognizing clonal neoantigens, and provided durable clinical benefits 51 and 52 . In the case of EBV+ patients, PDL1/2 overexpression and immune pathway activation signatures strongly justify targeting these molecules/pathways via immunotherapy, as they are most likely good candidates of immune response reactivation. Thus, the identification of tumors belonging to these two subgroups might prove fruitful in selecting candidates for immunotherapy, as it has been fairly effective for different neoplastic malignancies 53 and 54 (Box 4). Unfortunately, no drug-targeted genomic alterations have been identified so far in the genomically stable subtype, and thus targeting this subtype remains a critical aim in this field of investigation. 


\section{Box 4.}

\section{Clinician's Corner}

The prognosis of gastric cancer is poor and has not sufficiently improved in recent years.

Only two targeted therapies have been approved in advanced gastric cancer: Trastuzumab, in HER2-amplified patients, and Ramucirumab, targeting VEGFR2.

Clinical trials targeting other genetically altered tyrosine kinase receptors (such as EGFR, MET, FGFR2) have all failed. It is not yet clear if this is attributable to the fact that these molecules are not bona fide targets in this neoplasm or if clinical trials have not appropriately selected patients having the potential to respond to targeted therapies.

Two new molecular classifications of gastric cancer have been recently published. Although not completely overlapping, they can be of clinical help. The subtypes described by the TCGA classification, in fact, are associated with specific genetic 'druggable' alterations; the AGCR subtypes are associated with a different prognosis and thus could help physicians in modulating the intensity of a therapeutic regimen.

Recently, immunotherapy acting on checkpoint blockade has become an interesting option to potentially treat many tumors. Two gastric cancer subtypes (the TCGA EBV+ and MSI+ ones) show molecular characteristics suggesting a promising potential responsiveness to these immunotherapies.

Several cancer centers and health institutions all over the world have launched programs aimed at deriving in vitro and in vivo models from patient tumors. Studies performed in these models could help physicians understand the molecular basis of the clinical responses observed in patients from which the models have been derived. They could also identify new therapeutic strategies potentially effective in individual patients.

\section{Concluding Remarks}

As personalized medicine relies on tailoring patient treatments, based on molecular characteristics of a given disease, the two reports recently published by the TCGA and ACRG groups represent an important advance in the field of gastric cancer. Given that the use of targeted therapies in gastric tumors is much less common than in other cancers, the reported data provide a critical starting point to design more appropriate clinical trials based on the principles of precision medicine. To eventually identify bona fide molecular targets and develop solid therapeutic approaches, accurate patient selection for clinical trials is cogent. Moreover, recently available preclinical models (including tumor-derived cell lines, organoids, and PDXs) and the design of co-clinical trials (useful to address drug responses in PDXs, interrogating novel combination strategies to overcome emergent resistance) should be used to assist the clinical development of therapeutics (see Outstanding Questions and Box 4). It is thus desirable that acquired molecular knowledge derived from genomic, transcriptomics, and other 'omic' technologies be envisaged as a solid starting point to substantially improve the efficacy of gastric cancer treatment.

Can the identification of the molecular landscape of gastric cancer help us define positive and negative predictors of response to Trastuzumab? Is Trastuzumab the best anti-HER2 therapy in gastric cancer? Can Trastuzumab only target gastric tumors displaying HER2 amplification or can it also target tumors bearing HER2 mutations?

Preclinical models have shown the effectiveness of targeting EGFR, MET, and FGFR2 in gastric cancer cells. As clinical trials targeting these receptor tyrosine kinases have yielded negative results, can we still consider them as relevant intervention targets in gastric cancer?

PIK3CA is frequently mutated in gastric cancer and PI3K inhibition has shown clinical benefit in other malignancies. Can we accurately identify the PI3K mutations that render this kinase an oncogenic driver? What is the best strategy to effectively target PI3K in gastric cancer? 
Are the EBV+ and MSI+ subtypes (respectively showing high expression levels of PDL1/2 and high neoantigen burden) solid candidates for immunotherapy?

Can molecular profiling help us identify originating 'trunk mutations' in gastric cancer?

How do gastric cancer molecular profiles compare with other malignancies (e.g., colorectal cancer), and what can we learn from these comparisons? Are there common pathways and genes being upregulated/downregulated, and can this knowledge be applied to achieve more effective targeting/drug development?

How does genetic intratumor heterogeneity impact the efficacy of targeted therapies in gastric cancer? How does genetic heterogeneity in primary versus metastatic gastric tumors sustain the occurrence of therapeutic resistance?

What are the most optimal ways to use PDXs to facilitate the identification of new therapeutic targets in gastric cancer?

\section{Acknowledgments}

This work was supported by the Associazione Italiana per la Ricerca sul Cancro (AIRC) IG-15464 grant to S.G.

\section{References}

1. J. Ferlay, et al. Cancer incidence and mortality worldwide: sources, methods and major patterns in GLOBOCAN 2012. Int. J. Cancer, 136 (2015), pp. E359-E386

2. A. Jemal, et al. Global cancer statistics. CA Cancer J. Clin., 61 (2011), pp. 69-90

3. S. Nagini. Carcinoma of the stomach: a review of epidemiology, pathogenesis, molecular genetics and chemoprevention. World J. Gastrointest. Oncol., 4 (2012), pp. 156-169

4. C. Jackson, et al. Gastric cancer: ESMO clinical recommendations for diagnosis, treatment and follow-up. Ann. Oncol., 20 (Suppl. 4) (2009), pp. 34-36

5. K.D. Crew, A.I. Neugut. Epidemiology of gastric cancer. World J. Gastroenterol., 12 (2006), pp. 354-362

6. G. Corso, et al. History, pathogenesis, and management of familial gastric cancer: original study of John XXIII's family Biomed. Res. Int., 2013 (2013), p. 385132

7. D. Shibata, L.M. Weiss. Epstein-Barr virus-associated gastric adenocarcinoma. Am. J. Pathol., 140 (1992), pp. 769774

8. P. Lauren. The two histological main types of gastric carcinoma: diffuse and so-called intestinal-type carcinoma. An attempt at a histo-clinical classification. Acta Pathol. Microbiol. Scand., 64 (1965), pp. 31-49

9. N. Deng, et al. A comprehensive survey of genomic alterations in gastric cancer reveals systematic patterns of molecular exclusivity and co-occurrence among distinct therapeutic targets. Gut, 61 (2012), pp. 673-684

10. S. Kiyose, et al. Detection of kinase amplifications in gastric cancer archives using fluorescence in situ hybridization Pathol. Int., 62 (2012), pp. 477-484

11. The Cancer Genome Atlas Research Network. Comprehensive molecular characterization of gastric adenocarcinoma. Nature, 513 (2014), pp. 202-209 
12 R. Cristescu, et al. Molecular analysis of gastric cancer identifies subtypes associated with distinct clinical outcomes Nat. Med., 21 (2015), pp. 449-456

13. Y.J. Bang, et al. Trastuzumab in combination with chemotherapy versus chemotherapy alone for treatment of HER2positive advanced gastric or gastro-oesophageal junction cancer (TOGA): a phase 3, open-label, randomised controlled trial. Lancet, 376 (2010), pp. 687-697

14. C.S. Fuchs, et al. Ramucirumab monotherapy for previously treated advanced gastric or gastro-oesophageal junction adenocarcinoma (REGARD): an international, randomised, multicentre, placebo-controlled, phase 3 trial. Lancet, 383 (2014), pp. 31-39

15. H. Wilke, et al. Ramucirumab plus paclitaxel versus placebo plus paclitaxel in patients with previously treated advanced gastric or gastro-oesophageal junction adenocarcinoma (RAINBOW): a double-blind, randomised phase 3 trial. Lancet Oncol., 15 (2014), pp. 1224-1235

16. T. Satoh, et al. Lapatinib plus paclitaxel versus paclitaxel alone in the second-line treatment of HER2-amplified advanced gastric cancer in Asian populations: TyTAN-a randomized, phase III study. J. Clin. Oncol., 32 (2014), pp. 2039-2049

17. J.R. Hecht, et al. Lapatinib in combination with capecitabine plus oxaliplatin in human epidermal growth factor receptor 2-positive advanced or metastatic gastric, esophageal, or gastroesophageal adenocarcinoma: TRIO013/LOGiC-a randomized Phase III trial. J. Clin. Oncol., 34 (2016), pp. 443-451

18. Y.-K. Kang, et al. A randomized, open-label, multicenter, adaptive Phase $2 / 3$ study of trastuzumab emtansine (TDM1) versus a taxane (TAX) in patients (pts) with previously treated HER2-positive locally advanced or metastatic gastric/gastroesophageal junction adenocarcinoma (LA/MGC/GEJC). J. Clin. Oncol., 34 (Suppl. 4S;abstract 5) (2016)

19. A. Ohtsu, et al. Bevacizumab in combination with chemotherapy as first-line therapy in advanced gastric cancer: a randomized, double-blind, placebo-controlled phase III study. J. Clin. Oncol., 29 (2011), pp. 3968-3976

20. I.B. Weinstein. Cancer. Addiction to oncogenes-the Achilles heal of cancer. Science, 297 (2002), pp. 63-64

21. I.B. Weinstein, A.K. Joe. Mechanisms of disease: oncogene addiction-a rationale for molecular targeting in cancer therapy. Nat. Clin. Pract. Oncol., 3 (2006), pp. 448-457

22. C. Gomez-Martin, et al. Level of HER2 gene amplification predicts response and overall survival in HER2-positive advanced gastric cancer treated with trastuzumab. J. Clin. Oncol., 31 (2013), pp. 4445-4452

23. A. Bertotti, et al. A molecularly annotated platform of patient-derived xenografts ('xenopatients') identifies HER2 as an effective therapeutic target in cetuximab-resistant colorectal cancer. Cancer Discov., 1 (2011), pp. 508-523

24. S.M. Kavuri, et al. HER2 activating mutations are targets for colorectal cancer treatment. Cancer Discov., 5 (2015), pp. 832-841

25. A. Sartore-Bianchi, et al. Dual-targeted therapy with trastuzumab and lapatinib in treatment-refractory, KRAS codon 12/13 wild-type, HER2-positive metastatic colorectal cancer (HERACLES): a proof-of-concept, multicentre, open-label, phase 2 trial. Lancet Oncol. (2016) Published online April 20, 2016 http://dx.doi.org/10.1016/S1470-2045(16)00150-9

26. L. Xie, et al. FGFR2 gene amplification in gastric cancer predicts sensitivity to the selective FGFR inhibitor AZD4547. Clin. Cancer Res., 19 (2013), pp. 2572-2583

27. P.R. Gavine, et al. Volitinib, a potent and highly selective c-Met inhibitor, effectively blocks c-Met signaling and growth in c-MET amplified gastric cancer patient-derived tumor xenograft models. Mol. Oncol., 9 (2015), pp. 323-333

28. S. Li, et al. Maternal embryonic leucine zipper kinase serves as a poor prognosis marker and therapeutic target in gastric cancer. Oncotarget, 7 (2016), pp. 6266-6280 
29. H. Gao, et al. High-throughput screening using patient-derived tumor xenografts to predict clinical trial drug response. Nat. Med., 21 (2015), pp. 1318-1325

30. Y.Y. Choi, et al. Establishment and characterisation of patient-derived xenografts as paraclinical models for gastric cancer. Sci. Rep., 6 (2016), p. 22172

31. T.S. Kim, et al. Novel gastric cancer cell lines established from diffuse type gastric cancer patients for potential subgroup-specific therapy. Proc. AACR 2016, 57 (A) (2016) abstract 139

32. A. Fatehullah, et al. Organoids as an in vitro model of human development and disease. Nat. Cell Biol., 18 (2016), pp. 246-254

33. X. Li, et al. Oncogenic transformation of diverse gastrointestinal tissues in primary organoid culture. Nat. Med., 20 (2014), pp. 769-777

34. K. Wang, et al. Whole-genome sequencing and comprehensive molecular profiling identify new driver mutations in gastric cancer. Nat. Genet., 46 (2014), pp. 573-582

35. L.D. Nadauld, et al. Metastatic tumor evolution and organoid modeling implicate TGFBR2 as a cancer driver in diffuse gastric cancer. Genome Biol., 15 (2014), p. 428

36. N. Sachs, H. Clevers. Organoid cultures for the analysis of cancer phenotypes. Curr. Opin. Genet. Dev., 24 (2014), pp. 68-73

37. A.V. Lee, N.E. Davidson. Breast cancer in 2013: genomics, drug approval, and optimal treatment duration. Nat. Rev. Clin. Oncol., 11 (2014), pp. 71-72

38. F. Lordick, et al. Capecitabine and cisplatin with or without cetuximab for patients with previously untreated advanced gastric cancer (EXPAND): a randomised, open-label phase 3 trial. Lancet Oncol., 14 (2013), pp. 490-499 |

39. T. Waddell, et al. Epirubicin, oxaliplatin, and capecitabine with or without panitumumab for patients with previously untreated advanced oesophagogastric cancer (REAL3): a randomised, open-label phase 3 trial. Lancet Oncol., 14 (2013), pp. 481-489

40. M.A. Shah, et al. Phase II study evaluating 2 dosing schedules of oral foretinib (GSK1363089), cMET/VEGFR2 inhibitor, in patients with metastatic gastric cancer. PLoS ONE, 8 (2013), p. e54014

41. A.M. Shah, et al. METGastric: a phase III study of onartuzumab plus mFOLFOX6 in patients with metastatic HER2negative (HER2-) and MET-positive (MET + ) adenocarcinoma of the stomach or gastroesophageal junction (GEC). J. Clin. Oncol., 33 (Suppl) (2015) abstract 4012

42. D. Cunningham, et al. Phase III, randomized, double-blind, multicenter, placebo (P)-controlled trial of rilotumumab (R) plus epirubicin, cisplatin and capecitabine (ECX) as first-line therapy in patients (pts) with advanced MET-positive (pos) gastric or gastroesophageal junction (G/GEJ) cancer: RILOMET-1 study. J. Clin. Oncol., 33 (Suppl) (2015) abstract 4000

43. T. Doi, et al. A phase 3, multicenter, randomized, double-blind, placebo-controlled study of rilotumumab in combination with cisplatin and capecitabine (CX) as first-line therapy for Asian patients (pts) with advanced MET-positive gastric or gastroesophageal junction (G/GEJ) adenocarcinoma: the RILOMET-2 trial. J. Clin. Oncol., 33 (Suppl. 3) (2015) abstract TPS226

44. Y.-J. Bang, et al. A randomized, open-label phase II study of AZD4547 (AZD) versus paclitaxel (P) in previously treated patients with advanced gastric cancer (AGC) with fibroblast growth factor receptor 2 (FGFR2) polysomy or gene amplification (amp): SHINE study. J. Clin. Oncol., 33 (Suppl.) (2015) abstract 4014

45. K. Suda, et al. Reciprocal and complementary role of MET amplification and EGFR T790 M mutation in acquired resistance to kinase inhibitors in lung cancer. Clin. Cancer Res., 16 (2010), pp. 5489-5498 
46. A. Ohtsu, et al. Everolimus for previously treated advanced gastric cancer: results of the randomized, double-blind, phase III GRANITE-1 study. J. Clin. Oncol., 31 (2013), pp. 3935-3943

47. R.K. Ramanathan, et al. Phase 2 study of MK-2206, an allosteric inhibitor of AKT, as second-line therapy for advanced gastric and gastroesophageal junction cancer: a SWOG cooperative group trial (S1005). Cancer, 121 (2015), pp. 2193-2197

48. M. Greaves. Leukaemia 'firsts' in cancer research and treatment. Nat. Rev. Cancer, 16 (2016), pp. 163-172

49. A. Desrichard, et al. Cancer neoantigens and applications for immunotherapy. Clin. Cancer Res., 22 (2016), pp. 807-812

50. T.N. Schumacher, R.D. Schreiber. Neoantigens in cancer immunotherapy. Science, 348 (2015), pp. 69-74

51. N. McGranahan, et al. Clonal neoantigens elicit T cell immunoreactivity and sensitivity to immune checkpoint blockade. Science, 351 (2016), pp. 1463-1469

52. Y. Xiao, G.J. Freeman. The microsatellite instable subset of colorectal cancer is a particularly good candidate for checkpoint blockade immunotherapy. Cancer Discov., 5 (2015), pp. 16-18

53. J.R. Brahmer, et al. Safety and activity of anti-PD-L1 antibody in patients with advanced cancer. N. Engl. J. Med., 366 (2012), pp. 2455-2465

54. E.J. Lipson, et al. Antagonists of PD-1 and PD-L1 in cancer treatment. Semin. Oncol., 42 (2015), pp. 587-600

55. J.I. Cohen, et al. Epstein-Barr virus: an important vaccine target for cancer prevention. Sci. Transl. Med., 3 (2011), p. $107 \mathrm{fs} 107$

56. C.M. Tsang, et al. Cyclin D1 overexpression supports stable EBV infection in nasopharyngeal epithelial cells. Proc. Natl. Acad. Sci. U.S.A., 109 (2012), pp. E3473-E3482

57. M. Hidalgo, et al. Patient-derived xenograft models: an emerging platform for translational cancer research. Cancer Discov., 4 (2014), pp. 998-1013

58. M. Gerlinger, et al. Intratumor heterogeneity and branched evolution revealed by multiregion sequencing. N. Engl. J. Med., 366 (2012), pp. 883-892

59. C. Swanton. Intratumor heterogeneity: evolution through space and time. Cancer Res., 72 (2012), pp. 4875-4882 